\title{
Ocular Pharmacokinetics of Brimonidine Drug Delivery System in Monkeys and Translational Modeling for Selection of Dose and Frequency in Clinical Trials
}

\author{
Mitalee Tamhane, Kenneth T. Luu, ${ }^{1}$ and Mayssa Attar \\ Allergan, an AbbVie company, Irvine, California
}

Received December 23, 2020; accepted June 28, 2021

\begin{abstract}
Brimonidine, a selective $\alpha_{2}$-adrenoceptor agonist, displays putative retinal cyto- and neuroprotective activity in vitro and in vivo. An intravitreal sustained-release brimonidine implant, Brimonidine Posterior Segment Drug Delivery System (brimonidine DDS), allowing targeted drug delivery to the retina has been developed for potential clinical application. This study evaluates the in vivo posterior segment pharmacokinetics of brimonidine DDS implant in the monkey eye and applies translational pharmacokinetic modeling to predict tissue exposure in the human eye. Anesthetized cynomolgus monkeys received a single intravitreal injection of brimonidine DDS $400 \mu \mathrm{g}$ implant before removal of study eyes at days $7,30,60,92,120$, and 150 postimplant (three to four animals per time point) for assay of brimonidine in aqueous humor, vitreous, and retina samples. Brimonidine concentrations in the human eye were modeled using a linear, three-compartment model assuming bidirectional distribution to/from the aqueous humor and retina and elimination from the aqueous humor. Monkey tissue volumes were scaled up to human values; intercompartmental and elimination rate constants were assumed to be identical in the two species. Modeling and simulations were performed using NONMEM v. 7.3, R 3.5.1. Brimonidine exposure was highest in the monkey
\end{abstract}

vitreous and retina; concentrations in the central (macula) and peripheral retina were maintained at high levels $(>100 \mathrm{ng} / \mathrm{g})$ for 3 to 4 months. Simulated brimonidine concentration-time profiles in human macula indicated that brimonidine DDS $400 \mu \mathrm{g}$ implant would deliver effective drug concentrations $(20.7-82.2 \mathrm{ng} / \mathrm{g}$, based on animal pharmacology) for approximately 3 months. Accordingly, administration of the $400 \mu \mathrm{g}$ implant at 3-month intervals is recommended.

\section{SIGNIFICANCE STATEMENT}

Brimonidine, an $\alpha_{2}$-adrenoceptor agonist, is cyto- and neuroprotective in animal models of retinal/optic nerve injury. Brimonidine Posterior Segment Drug Delivery System (brimonidine DDS) is an intravitreal sustained-release implant with potential ophthalmological applications. This study explores the pharmacokinetics of brimonidine DDS $400 \mu \mathrm{g}$ implant in the monkey eye and uses compartmental modeling to predict human ocular tissue exposure. Targeted retinal brimonidine delivery from vitreous was demonstrated in monkeys. Simulated tissue concentration-time profiles indicated persistence of pharmacologically effective brimonidine concentrations for $\approx 3$ months in human retina.

\section{Introduction}

Brimonidine is a highly selective $\alpha_{2}$-adrenergic receptor agonist that is currently approved in the United States and Europe for the treatment of open-angle glaucoma and ocular hypertension. In addition to its intraocular pressure-lowering effect, experimental studies have demonstrated that brimonidine possesses cyto- and neuroprotective activity in a variety of animal models of retinal and optic nerve injury, including

This study was supported by Allergan plc, Irvine, CA (before its acquisition by AbbVie).

All authors are current or former full-time employees and stockholders of AbbVie, Inc.

${ }^{1}$ Current affiliation: AnaptysBio Inc., San Diego, CA.

Parts of this analysis were accepted as an Abstract submission for presentation at the Association for Research in Vision and Ophthalmology 2016 Annual Meeting, May 1-5, 2016, Seattle, WA

https://dx.doi.org/10.1124/jpet.120.000483. acute retinal ischemia (Donello et al., 2001; Lafuente et al., 2001; Vidal-Sanz et al., 2001; Lai et al., 2002), excitotoxic retinal injury (Galindo-Romero et al., 2016), blue light phototoxicity (Ortín-Martínez et al., 2014; Valiente-Soriano et al., 2019), chronic ocular hypertension (WoldeMussie et al., 2001; Hernández et al., 2008), and optic nerve crush (Yoles et al., 1999; Saylor et al., 2009). In a chronic progressive outer retinal degeneration model of geographic atrophy in monkeys, brimonidine prevented light-induced damage to retinal pigment epithelium and photoreceptors (Rajagopalan et al., 2019). The cyto- and neuroprotective effect of brimonidine is thought to be related to its enhancement of the ability of retinal neuronal cells to resist cellular stress, an effect possibly achieved through activation of cell-survival signaling pathways or interference with cytotoxic signaling (Peng et al., 1998; Wheeler et al., 2003; Saylor et al., 2009). Research indicates that the $\alpha_{2}$-adrenergic receptor is expressed throughout

ABBREVIATIONS: AUC, area under concentration-time curve; $\mathrm{AUC}_{0 \text {-tlast }}$ AUC from time 0 to the last quantifiable sampling time; $B L Q$, below limit of quantification; brimonidine DDS, Brimonidine Posterior Segment Drug Delivery System; OFV, objective function value; RPE, retinal pigment epithelium. 
the neurosensory retina (Woldemussie et al., 2007) and that its activation has cascading effects on signal pathways that block apoptosis (Wheeler et al., 2003). Brimonidine demonstrates cyto- and neuroprotective activity in vitro, reducing the toxic effects of hydroquinone exposure on human retinal pigment epithelium and retinal Muller cells and rendering them more resistant to injury (Ramírez et al., 2016). These data show that brimonidine has pharmacological properties consistent with protection of retinal cellular and neuronal function.

Clinical evidence of a potential cyto- and neuroprotective effect of brimonidine is provided by the Low-Pressure Glaucoma Treatment Study, in which topical brimonidine was found to reduce the risk of visual field progression compared with timolol in patients with normal-tension glaucoma despite the two drugs showing similar ocular hypotensive effect (Krupin et al., 2011). Additionally, the cyto- and neuroprotective effect of brimonidine has been explored in patients with age-related macular degeneration (Ferencz et al., 2005), geographic atrophy (Kuppermann et al., 2020), retinitis pigmentosa (Merin et al., 2008), diabetic retinopathy (Mondal et al., 2004; Simó et al., 2019), and acute nonarteritic anterior ischemic optic neuropathy (Wilhelm et al., 2006).

To exert a durable cyto- and neuroprotective effect, a drug should be delivered in adequate concentrations to the retina over an extended period. Topical brimonidine (brimonidine tartrate $0.2 \%$ ophthalmic solution) shows only limited posterior segment penetration, achieving vitreous concentrations of $\leq 5 \mathrm{ng} / \mathrm{ml}$ in the phakic human eye (Kent et al., 2001). Intravitreal administration enables targeted delivery of brimonidine to the retina. However, brimonidine undergoes rapid clearance after intravitreal injection, with a vitreous elimination halflife of 1.45 hours after single bolus administration (928 ng in $50-\mu \mathrm{l}$ injection volume) in the rabbit eye (unpublished Allergan data). Due to the short duration of ocular exposure, frequent intravitreal injections would be necessary to achieve sustained retinal tissue concentrations. To address these constraints, an intravitreal implant consisting of brimonidine $400 \mu \mathrm{g}$ free base in a slow-release polymer matrix - the sustained-release Brimonidine free-base Posterior Segment Drug Delivery System (brimonidine DDS; Allergan, an AbbVie company, Irvine, CA) - has been developed for potential clinical application in the treatment of geographic atrophy and for cyto- and neuroprotection in glaucoma. The implant evaluated in this study offers the advantage over earlier prototypes of providing a higher drug load, faster drug release, and more rapid matrix bioerosion, resulting in higher ocular tissue exposure.

Despite interspecies differences in ocular anatomic and physiologic parameters, animal studies can provide reliable estimates of vitreous pharmacokinetics in the human eye (Del Amo and Urtti, 2015). For this purpose, monkeys are commonly used to evaluate ocular pharmacokinetic profiles (Gaudreault et al., 2005; Chang-Lin et al., 2011; Shen et al., 2014; Niwa et al., 2015). The objectives of this study were 1) to evaluate the in vivo posterior segment pharmacokinetics of brimonidine DDS $400 \mu \mathrm{g}$ implant in the monkey eye, 2) to perform compartmental modeling of the monkey pharmacokinetic data to enable translation to humans, and 3) to use the translational model to predict vitreous and macula exposure in the human eye and select an appropriate dosage regimen for clinical testing.

\section{Materials and Methods}

Animal Experiments. The in vivo portion of this study was conducted at Covance Laboratories Inc. (Madison, WI) in compliance with the Animal Welfare Act (Regulations 9 Code of Federal Regulations Parts 1, 2, and 3) and the Association for Research in Vision and Ophthalmology Statement for the Use of Animals in Ophthalmic and Vision Research.

Male cynomolgus monkeys weighing approximately 2.3 to $3.7 \mathrm{~kg}$ were obtained from Covance Research Products Inc. (Denver, PA, and Alice, TX). The animals were housed in a temperature-controlled facility with a 12-hour light/dark cycle with unrestricted access to food and water and were acclimated to study conditions for 4 weeks.

In preparation for dosing with brimonidine DDS $400 \mu \mathrm{g}$ implant, monkeys were lightly anesthetized with intramuscular ketamine $10 \mathrm{mg} / \mathrm{kg}$ and dexmedetomidine $0.025 \mathrm{mg} / \mathrm{kg}$, with additional anesthesia provided as needed. Ocular preparation for intravitreal injection involved administration of one or two drops of a topical ophthalmic anesthetic (proparacaine hydrochloride $0.5 \%$ ) followed by a broadspectrum ophthalmic microbicide (povidone-iodine 5\% solution; Betadine). After 2 to 3 minutes, study eyes were irrigated with sterile saline, and a drop of topical ophthalmic anesthetic (lidocaine hydrochloride $2 \%$ ) was applied, followed by an ophthalmic fluoroquinolone antibiotic ointment for infection prophylaxis. A 25-gauge needle and preloaded brimonidine DDS applicator was used to deliver a single intravitreal injection of brimonidine DDS $400 \mu \mathrm{g}$ implant (Allergan, an AbbVie company, Irvine, CA) to the study eye. Injections were performed in the dorsotemporal quadrant, approximately $2.5 \mathrm{~mm}$ posterior to the limbus, through the sclera and pars plana. The bevel of the needle was directed downward and posteriorly to avoid the lens. Brimonidine DDS $400 \mu \mathrm{g}$ implant was deployed in the center of the vitreous by depressing an actuator button on the applicator.

At predetermined time intervals over 5 months postimplant (days $7,30,60,92,120$, and 150), three or four animals per time point were euthanized via exsanguination under sodium pentobarbital anesthesia. Blood samples (approximately $5 \mathrm{ml}$ ) were collected via cardiac puncture at the time of sacrifice, stored in K3 EDTA-coated tubes on wet ice, and centrifuged to obtain plasma. Study eyes were enucleated at the time of sacrifice, aqueous humor was removed, and the eyes were then flash-frozen in liquid nitrogen before collection (within 2 days) of vitreous, retina, and choroid with retinal pigmented epithelium (RPE). Vitreous was collected together with the implant remnants, and the latter were separated from the defrosted vitreous before assay of vitreous drug concentrations. Retina samples consisted of 8-mm-diameter circular punch biopsies obtained from the macula (central retina) and from the remaining retinal tissue (peripheral retina).

External ophthalmic examination, slit-lamp biomicroscopy of the adnexa and anterior eye, and indirect ophthalmoscopy of the fundus were conducted in ketamine-anesthetized monkeys prior to intravitreal dosing and at days $3,14,92,180$, and 270 postdose by a boardcertified veterinary ophthalmologist.

Bioanalysis. Concentrations of brimonidine in plasma and ocular tissue samples were assayed by liquid chromatography-tandem mass spectrometry using an API 3000 or API 5000 triple-quadrupole spectrometer (AB Sciex) interfaced with a high-performance liquid chromatography system (Shimadzu Scientific Instruments, Columbia, MD) and autosampler (Shimadzu Scientific Instruments, Columbia, MD; Perkin-Elmer). High-performance liquid chromatography was performed on a Synergi Polar-RP column $(2.0 \times 50 \mathrm{~mm}, 4 \mu \mathrm{m}$; Phenomenex Corp.) using mobile phase A with $0.1 \%$ formic acid in 70:30 acetonitrile:water and mobile phase $\mathrm{B}$ with $0.1 \%$ ammonium hydroxide in 70:30 acetonitrile:water at flow rates of 0.3 or $0.5 \mathrm{ml} / \mathrm{min}$. Mass spectrometric detection was performed using multiple reaction monitoring scan mode with precursor/product ion pairs mass-to-charge ratio $(\mathrm{m} / \mathrm{z}) 292 \rightarrow \mathrm{m} / \mathrm{z} 212$ for brimonidine and $\mathrm{m} / \mathrm{z} 296 \rightarrow \mathrm{m} / \mathrm{z} 216$ for brimonidine-d4. Assay ranges for brimonidine in plasma and ocular tissues were $0.05-10.0 \mathrm{ng} / \mathrm{ml}$ (plasma), $0.1-50.0 \mathrm{ng} / \mathrm{ml}$ (aqueous 
humor), $0.25-200.0 \mathrm{ng} / \mathrm{ml}$ (vitreous), and 0.1-100.0 ng (retina and choroid-RPE).

Pharmacokinetic Analysis. Noncompartmental analysis was used to determine the following pharmacokinetic parameters for brimonidine: maximum concentration, time to maximum concentration, and area under the concentration-time curve from time 0 to the last quantifiable sampling time $\left(\mathrm{AUC}_{0 \text {-tlast }}\right)$. For $\mathrm{AUC}_{0 \text {-tlast, mean and }}$ S.E.M. values were calculated using the composite AUC calculation available in Watson version 7.3 software (Waltham, MA). Mean (S.D. and S.E.) tissue concentrations of brimonidine were calculated for each time point using Watson version 7.3 software. Within each data group, if $\leq 50 \%$ of individual readings were below the limit of quantification (BLQ), a value of zero was substituted for BLQ readings, and these substituted values were included in the calculation. If $>50 \%$ of readings were BLQ, the mean value was reported as "not calculable."

Pharmacokinetic Modeling and Human Dose Prediction. Brimonidine concentrations in human aqueous humor, vitreous, and retina (macula) were modeled using a linear, three-compartment model (Fig. 1). As shown in the figure, the model assumes that brimonidine undergoes slow release from the implant into the vitreous, and is distributed bidirectionally to and from the aqueous humor and retina. Different structural models were tested to fit the data in all three tissues: 1) a model involving drug elimination from both the retina and the aqueous humor and 2) a model with drug elimination occurring from either the retina or the aqueous humor. Based on estimates of the objective function value (OFV) of the various models, the model involving drug elimination from the aqueous humor was chosen (see Results and Discussion sections). A bioavailability factor was included to offset the tendency of the model to overpredict aqueous humor concentrations and to account for potential binding of brimonidine to melanin (see Discussion). The device release rate (zero-order) was previously determined from in vitro experiments to be $5.9 \mu \mathrm{g} / \mathrm{day}$; the same value was assumed to apply under in vivo conditions and was fixed in the model. Volume of drug distribution was fixed to the specific anatomic characteristics (weight or volume) of each tissue (Table 1). This approach allows for interspecies scaling, substituting human for monkey tissue volumes. Intercompartmental and elimination rate constants were assumed to be identical for monkeys and humans. Once the model was scaled to the human eye, simulations were performed to predict a suitable dose and regimen for testing in the clinic.

Modeling and simulations were performed using NONMEM v. 7.3, $R$ 3.5.1. The data were kept as naïve pool for model fitting. The firstorder conditional estimation method was implemented in NONMEM. The final model applied additive and proportional residual error model. The model was fitted to concentration data for the aqueous humor, vitreous, and retina simultaneously to obtain parameter estimates. Concentrations below the limit of detection were treated with the M5 method (dividing the BLQ by 2). Parameter precision was obtained by importance sampling following first-order conditional estimation.

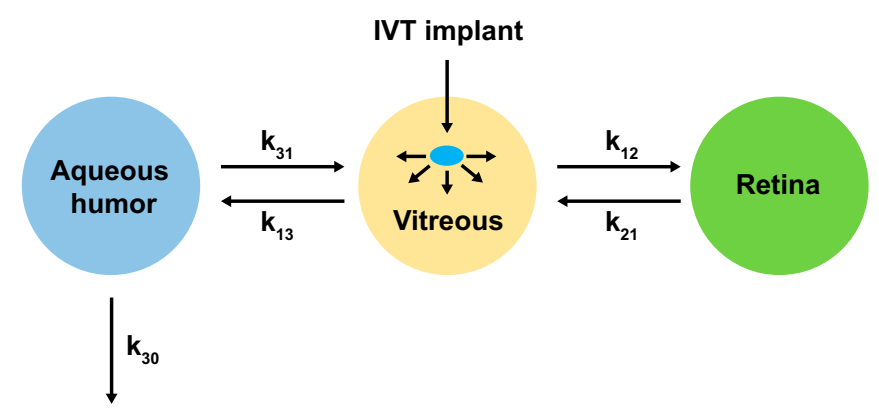

Fig. 1. Schematic presentation of the pharmacokinetic model used to describe the monkey data. Parameter descriptions are provided in Table 3. IVT, intravitreal.

\section{Results}

A total of 22 monkeys (22 eyes) received the brimonidine DDS $400 \mu \mathrm{g}$ implant, and safety and pharmacokinetic data were obtained from 22 and 18 eyes, respectively. Ophthalmic examination indicated that the intravitreal implant was well tolerated over 9 months of follow-up. All animals appeared healthy with no overt signs of toxicity relating to the implant. In a minority of animals, the implant elicited a mild and transient increase in vitreous cell numbers that subsided within approximately 90 days. Injection-related effects (subconjunctival hemorrhage at the injection site, mildly degraded view of the fundus, and fibrin/blood on the tip of the implant or in the vitreous) were short-lived, being evident only at day 3 postdose.

Pharmacokinetics of Brimonidine DDS in Monkeys. Noncompartmental pharmacokinetic parameters for brimonidine in each tissue are summarized in Table 2. Brimonidine exposure was highest in the vitreous and retina (macula and periphery), with lower concentrations observed in the aqueous humor, indicating targeted retinal drug delivery from the vitreous. Mean brimonidine concentration peaked in the vitreous $(10,900 \mathrm{ng} / \mathrm{ml})$ and peripheral retina $(12,200 \mathrm{ng} / \mathrm{g})$ at 60 days postimplant and in the central retina $(714 \mathrm{ng} / \mathrm{g})$ at 30 days postimplant. Brimonidine concentrations in the retina and vitreous were maintained at high levels $(>100 \mathrm{ng} / \mathrm{g}$ and $>1000 \mathrm{ng} / \mathrm{ml}$, respectively) for the first 2 or 3 months postimplant before falling sharply to $<10 \mathrm{ng} / \mathrm{g}$ and $<1 \mathrm{ng} / \mathrm{ml}$, respectively, at 5 months. Brimonidine concentrations in retina were sustained for longer than those in the vitreous, remaining above $30 \mathrm{ng} / \mathrm{g}$ for the first 4 months postimplant compared with $<2 \mathrm{ng} / \mathrm{ml}$ at the same time point for vitreous. Brimonidine concentrations in macula remained quantifiable for at least 120 days postimplant. Systemic drug exposure was negligible after intravitreal administration of brimonidine DDS $400 \mu \mathrm{g}$ implant, with plasma brimonidine concentrations remaining below the limit of quantification $(0.05 \mathrm{ng} / \mathrm{ml})$ at all time points.

Pharmacokinetic Modeling of Monkey Data. The final model was a three-compartment, linear model with a single exit rate constant coming from the aqueous humor compartment $(\mathrm{OFV}=549)$. Adding a second exit compartment from the retina did not improve the fit or alter the OFV but resulted in inclusion of an additional parameter. The model with elimination occurring solely from the retina produced a higher OFV (562). The final model was selected on the basis of having the lowest OFV and fewest parameters of those models evaluated. The final model parameters are summarized in Table 3, and a schematic of the model is presented in Fig. 1. Addition of the bioavailability factor to the model improved the OFV by $>100$ points. Differential rates of drug distribution from vitreous to retina and from retina to vitreous provided optimal data fit and were adopted for the final model. Both additive and proportional (constant coefficient of variation) error models were tested to capture the residual error in concentration data for vitreous and retina. The final model had both additive and proportional error structures. Figure 2 presents the final model fit to the pharmacokinetic data in monkeys.

The volume or weight of the ocular tissues were changed from the values reported for monkeys to those reported for 
TABLE 1

Ocular tissue volumes and/or weights used to develop the pharmacokinetic model

\begin{tabular}{lcr} 
& \multicolumn{1}{c}{ Cynomolgus Monkey } & Human \\
\hline Vitreous (ml) & $1.8-2.0$ (used 1.8) (Kaufman et al., 1981) & 4.0 (Weir and Collins, 2010) \\
Retina (g) & 0.073 (Struble et al., 2014) & 0.326 (Feke et al., 1989) \\
Aqueous humor (ml) & 0.123 (Bill and Hellsing, 1965) & 0.310 (Weir and Collins, 2010) \\
\hline
\end{tabular}

Values were fixed in either the monkey model or scaled-up human model.

humans. Simulated brimonidine concentration-time profiles in human aqueous humor, vitreous, and retina (macula) are shown in Fig. 3. The brimonidine DDS $400 \mu \mathrm{g}$ implant is anticipated to deliver pharmacologically effective drug concentrations in the range of 20.7 to $82.2 \mathrm{ng} / \mathrm{g}$ (based on internal data generated in animal pharmacology models) to the human retina for approximately 3 months. These simulated brimonidine concentrations $(\sim 100 \mathrm{ng} / \mathrm{g})$ in human retina are 160 -fold higher than the in vitro $\mathrm{EC}_{50}$ value at the human $\alpha_{2^{-}}$ adrenergic receptor $(0.6 \mathrm{ng} / \mathrm{ml}$ or $2 \mathrm{nM})$. For estimating potential efficacious in vivo levels in humans, we assumed a multiple of 10 - to 100 -fold over the in vitro $\mathrm{EC}_{50}$ value to account for in vitro to in vivo translation. The "in vivo" efficacious concentrations generated in animal models fall within this multiple and hence were used as target levels.

\section{Discussion}

Intravitreal injection is a widely accepted route of administration for ensuring targeted drug delivery to the posterior segment of the eye. This route is approved for administration of anti-vascular endothelial growth factor therapy (pegaptanib, ranibizumab, and aflibercept) for choroidal neovascularization caused by "wet" age-related macular degeneration and also for corticosteroid treatments (triamcinolone acetonide) for intraocular inflammation (Novack, 2009). In addition to the above treatments, which are given as solution formulations, dexamethasone intravitreal implant (Ozurdex) is approved for treatment of posterior segment uveitis, retinal vein occlusion, and diabetic macular edema. In the case of small molecules, which usually exhibit a vitreous half-life of several hours, the implant delivery system ensures sustained drug delivery to the target tissue for long periods, thereby minimizing the frequency of injections. Brimonidine DDS, an intravitreal implant consisting of $400 \mu \mathrm{g}$ brimonidine free base loaded in a slowrelease polymer matrix, was developed to achieve pharmacologically effective concentrations in the retina for prolonged periods.
The in vivo study in monkeys was performed to characterize the ocular pharmacokinetics of single-dose brimonidine DDS $400 \mu \mathrm{g}$ implant after intravitreal administration. Findings indicated that brimonidine concentrations in the central retina (macula) and peripheral retina were maintained at high levels $(>100 \mathrm{ng} / \mathrm{g}$ ) for 3 to 4 months in this species.

The monkey is a well established model for studying the pharmacokinetics of intravitreally administered drugs (Short, 2008) and allows simultaneous sampling of ocular tissues at serial time points in different animals. The monkey eye resembles the human eye closely with regard to anatomy and physiology, including the presence of a macula, but it too has a small vitreous cavity $(2 \mathrm{ml})$, which needs to be taken into consideration when extrapolating pharmacokinetic findings. Since the monkey is an appropriate translational species with regard to macular pharmacokinetics, monkey data were used to predict human aqueous humor, vitreous, and macula concentrations using pharmacokinetic modeling. Aqueous humor and vitreous are the most commonly sampled matrices in humans to predict pharmacokinetics as well as biomarker response to correlate with efficacy endpoints for retinal disorders. Therefore, predicting concentrations in both human aqueous humor and vitreous, in addition to the target tissue, is of value. In building our pharmacokinetic model, we used brimonidine concentrations from retina central punch (macula) rather than peripheral or total retina, since the macula represents the key site of action of brimonidine.

Brimonidine has been shown to bind reversibly, and with high affinity, to ocular melanin in vitro (Tang-Liu et al., 1992) and to distribute preferentially to, and clear more slowly from, pigmented than nonpigmented ocular tissues in the monkey (Acheampong et al., 2002). We conjectured that the need to incorporate the bioavailability term to improve the fit of the aqueous humor data was attributable to extensive melanin binding, which lowers the concentration of free drug. Without integrating the bioavailability term, the model consistently overfitted the aqueous humor data. The discrepancy between the duration of brimonidine release from the implant ( 2 to 3

TABLE 2

Pharmacokinetic parameters of single-dose intravitreal brimonidine DDS $400 \mu \mathrm{g}$ implant in monkey eyes

Pharmacokinetic Parameter (Mean \pm S.D.)

\begin{tabular}{|c|c|c|c|c|}
\hline \multirow[b]{2}{*}{ Tissue } & \\
\hline & $\mathrm{T}_{\max }$ & $\mathrm{C}_{\max }$ & $\mathrm{AUC}^{a}$ & AUC Interval \\
\hline & days & $n g / m l$ or $n g / g$ & $n g \bullet d a y / m l$ or $n g \bullet d a y / g$ & days \\
\hline Aqueous humor & 120 & $253 \pm 307$ & $14,400 \pm 5550$ & $0-150$ \\
\hline Vitreous humor & 60 & $10,900 \pm 1140$ & $555,000 \pm 152,000$ & $0-150$ \\
\hline Retina-macula punch & 30 & $715 \pm 605$ & $40,200 \pm 12,800$ & $0-120$ \\
\hline Retina-remaining & 60 & $12,200 \pm 5100$ & $624,000 \pm 101,000$ & $0-150$ \\
\hline Choroid-RPE-punch & 92 & $25,100 \pm 16,000$ & $1,980,000 \pm 308,000$ & $0-150$ \\
\hline Choroid-RPE-remaining & 60 & $115,000 \pm 15,900$ & $11,800,000 \pm 626,000$ & $0-150$ \\
\hline
\end{tabular}

$\mathrm{T}_{\max }$, time to maximum mean concentration.

${ }^{a}$ Values are presented as mean \pm S.E. 
TABLE 3

Pharmacokinetic model parameters, their descriptions, estimates, and relative standard errors

\begin{tabular}{|c|c|c|c|}
\hline Model Parameter & Parameter Description & Estimate & RSE \\
\hline $\begin{array}{l}\mathrm{k}_{31} \\
\mathrm{k}_{13} \\
\mathrm{k}_{12} \\
\mathrm{k}_{21} \\
\mathrm{k}_{30} \\
\mathrm{~V}_{1} \\
\mathrm{~V}_{2} \\
\mathrm{~V}_{3} \\
\text { BA factor }\end{array}$ & $\begin{array}{c}\text { Linear distribution rate constant from the aqueous humor to the vitreous } \\
\text { Linear distribution rate constant from the vitreous to the aqueous humor } \\
\text { Linear distribution rate constant from the vitreous to the retina } \\
\text { Linear distribution rate constant from the retina to the vitreous } \\
\text { Elimination rate constant from the aqueous humor } \\
\text { Vitreous volume } \\
\text { Retina volume (tissue weight) } \\
\text { Aqueous humor volume } \\
\text { Aqueous humor bioavailability factor }\end{array}$ & $\begin{array}{l}1.44 \times 10^{-5} \text { day }^{-1} \\
0.402 \text { day }^{-1} \\
2.64 \times 10^{-4} \text { day }^{-1} \\
0.0644 \text { day }^{-1} \\
0.0222 \text { day }^{-1} \\
1.80 \mathrm{ml} \text { (fixed) } \\
0.0730 \mathrm{~g} \text { (fixed) } \\
0.123 \mathrm{ml} \text { (fixed) } \\
1.02 \times 10^{-4}\end{array}$ & $\begin{array}{c}\% \\
6.76 \times 10^{-5} \\
4.88 \times 10^{-6} \\
1.14 \times 10^{-5} \\
2.93 \times 10^{-6} \\
3.00 \times 10^{-5} \\
\text { NA } \\
\text { NA } \\
\text { NA } \\
3.05 \times 10^{-5}\end{array}$ \\
\hline
\end{tabular}

BA, bioavailability; NA, not applicable; RSE, relative standard error.

months) and the prolonged duration of retinal brimonidine concentrations ( $>5$ months) is consistent with drug-melanin binding in the retina. Likewise, the low levels of brimonidine seen in the vitreous between 90 and 150 days postimplant (i.e., after drug release from the implant has ceased) might be due to release of melanin-bound brimonidine and redistribution of free drug from retina to vitreous.

The model appeared to point to brimonidine being predominantly eliminated via the aqueous humor. Smaller and more lipophilic drugs are thought to be predominantly eliminated from the retina by crossing blood-retinal barriers, whereas larger or more hydrophilic drugs are eliminated from the anterior segment through aqueous humor outflow (Del Amo et al., 2017; Varela-Fernández et al., 2020). When we tested the model with elimination from both the aqueous humor and the retina, the estimated elimination rate constant from the aqueous humor $\left(\mathrm{k}_{30}\right)$ was $0.022 \mathrm{day}^{-1}$, whereas the retinal elimination rate constant $\left(\mathrm{k}_{20}\right)$ was $<1 \times 10^{-14} \mathrm{day}^{-1}$. Since retinal elimination was near zero and inclusion of this parameter did not improve the OFV, we decided to exclude retinal elimination from the final model. Although, in general, an empirical model such as this provides no proof of the mechanism of drug clearance, it is feasible that brimonidine might be eliminated predominantly from the aqueous humor on account of its highly hydrophilic nature and small molecular size, with the latter potentially allowing clearance through the chamber angle or Schlemm's canal (Agrahari et al., 2016).

Brimonidine's distribution to the posterior ocular tissues after intravitreal administration differs quantitatively from that reported after topical administration. In another study, twice-daily topical instillation of $0.5 \%\left[{ }^{14} \mathrm{C}\right]$ brimonidine tartrate solution ( $119 \mu \mathrm{g}$ of brimonidine) for 14 days (total dose $=$ $3332 \mu \mathrm{g}$ ) into monkey eyes resulted in the following order of drug distribution: choroid/retina > aqueous humor > vitreous (Acheampong et al., 2002). Mean brimonidine concentrations peaked in vitreous at 1 hour postdose $(0.071 \mu \mathrm{g}-\mathrm{Eq} / \mathrm{g})$ and in choroid/retina at 24 hours postdose $(30.6 \mu \mathrm{g}-\mathrm{Eq} / \mathrm{g})$, whereas estimated $\mathrm{AUC}_{0-90}$ days was $0.960 \mu \mathrm{g}$-Eq.day/g in vitreous and $1499.0 \mu \mathrm{g}-\mathrm{Eq} \cdot \mathrm{day} / \mathrm{g}$ in choroid/retina. (Data are reported in units of radioactivity since intact brimonidine was the major radioactive component in all tissues at all time points, accounting for $83 \%-98 \%$ of total radioactivity.) Thus, brimonidine exposure in vitreous after administration of brimonidine DDS $400 \mu \mathrm{g}$ was $>500$-fold higher than that achieved after topical administration of brimonidine tartrate $0.5 \%$ solution, despite the implant containing an 8-fold lower total brimonidine dose (total topical dose of brimonidine over 14 days $=$
$3332 \mu \mathrm{g})$. In the study of topically instilled $0.5 \%\left[{ }^{14} \mathrm{C}\right]$ brimonidine tartrate solution, retinal and choroidal tissues were not separated, and therefore total radioactivity or brimonidine concentrations were presented for combined retina/choroid. In our study with intravitreal brimonidine DDS, retina was separated from choroid-RPE and, furthermore, was subdivided into "retina central punch" (macula) and "retina periphery" (remaining retina). This difference in tissue sampling has a profound impact on estimates of brimonidine concentrations available for $\alpha_{2}$-adrenoceptor activation in the retina. Brimonidine binds strongly but reversibly to ocular melanin, and this can affect the drug's disposition and lead to overestimation of therapeutic drug levels in ocular tissue.

A study of the ocular distribution of topically administered $0.1 \%$ brimonidine tartrate ophthalmic solution $(35 \mu \mathrm{l})$ in the pigmented rabbit demonstrated that free brimonidine concentrations in retina/choroid were 100 -fold lower than total brimonidine concentrations (Shinno et al., 2017). Therefore, retina separated from choroid-RPE should provide a better estimate of brimonidine levels available for $\alpha_{2}$-adrenoceptor activation. As demonstrated in Fig. 2, after administration of brimonidine DDS $400 \mu \mathrm{g}$ implant, drug concentrations in monkey retina (macula), representing free brimonidine concentrations, are maintained above the pharmacologically relevant concentrations (20.7-82.2 ng/g; determined in pharmacology models of retinal degeneration) for 3 months postdose. Thus, comparison of posterior ocular tissue exposure after topical and intravitreal brimonidine implant administration suggests that the intravitreal delivery route provides higher posterior tissue concentrations and that these are sustained over a longer period, thereby avoiding the burden to the patient of daily eyedrop administration.

Our simulations of brimonidine concentration-time profiles in human aqueous humor, vitreous, and macula, based on pharmacokinetic modeling of the monkey data, suggest that, at a conservative estimate, the brimonidine DDS $400 \mu \mathrm{g}$ implant will provide sustained, pharmacologically effective drug concentrations in the human macula for approximately 3 months after intravitreal administration. Based on these model predictions, a dosage regimen of $400 \mu \mathrm{g}$, administered intravitreally every 3 months, was recommended for clinical trials.

Limitations to the data collection and model are worth noting. Firstly, we did not account for the location of the implant, which could impact drug distribution in the posterior tissues. Secondly, the device release rate was assumed to be constant over the duration of drug release and the same as the in vivo 
Aqueous humor

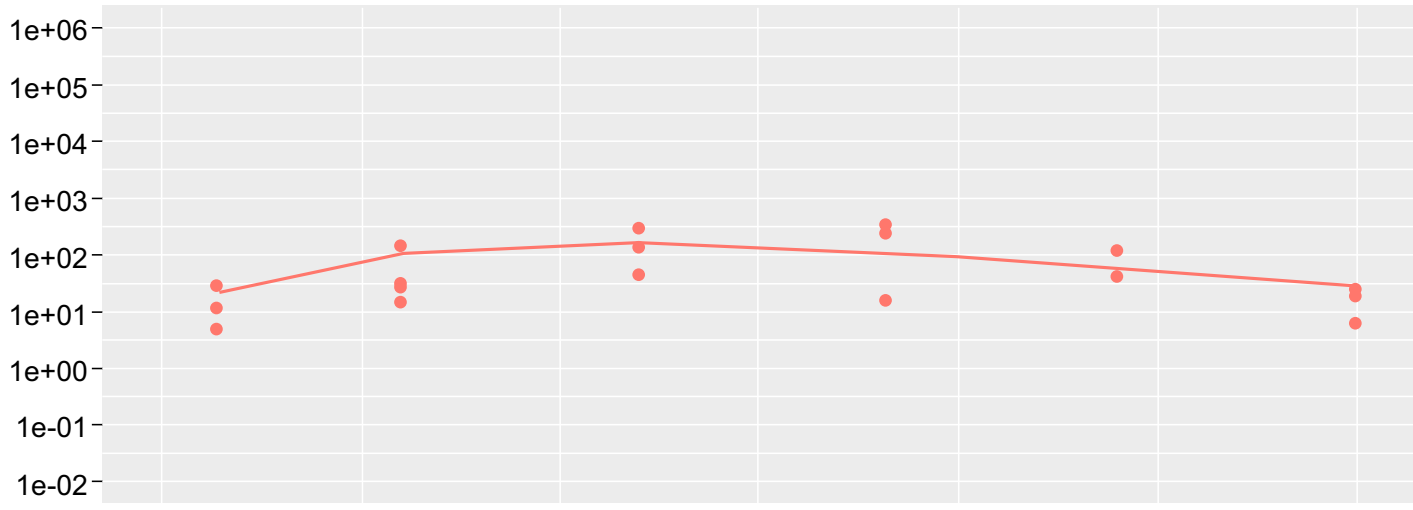

Retina (macula)

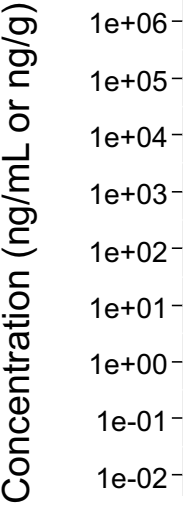

$1 e+06-$
$1 e+05^{-}$
$1 e+04^{-}$
$1 e+03^{-}$
$1 e+02^{-}$
$1 e+01-$
$1 e+00^{-}$
$1 e-01-$
$1 e-02-$

Vitreous

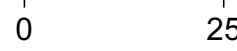

25

$\stackrel{1}{50}$

1
75

100

125

150

\section{Compartment}

Time (day)

Aqueous humor

- Retina (macula)

- - Vitreous

Fig. 2. Observed (symbols) and model-fitted (solid lines) brimonidine concentration-time profiles in monkey aqueous humor, vitreous, and retina (macula) after intravitreal administration of brimonidine DDS $400 \mu \mathrm{g}$ implant.

release rate. There could be differences in in vitro and in vivo release rates, which could impact the model predictions. Notwithstanding this, preliminary internal data (data not shown) indicated that there was good correlation between in vitro and in vivo release rates, and this assumption resulted in a reasonable fit of the model to the data. The model also assumed the intercompartmental and elimination rate constants to be identical for monkeys and humans since we are limited by the paucity of drug concentration data generated in human ocular tissues. Thirdly, since ocular pharmacokinetic sampling is a terminal procedure, serial pharmacokinetic data could not be collected from the same animal. Consequently, estimates of interindividual variability in pharmacokinetic parameters were unavailable, and the model was fitted to naïve pool data. Finally, the model assumes linear pharmacokinetics based on a single dose level. For this study, only the highest feasible 


\section{Aqueous humor}

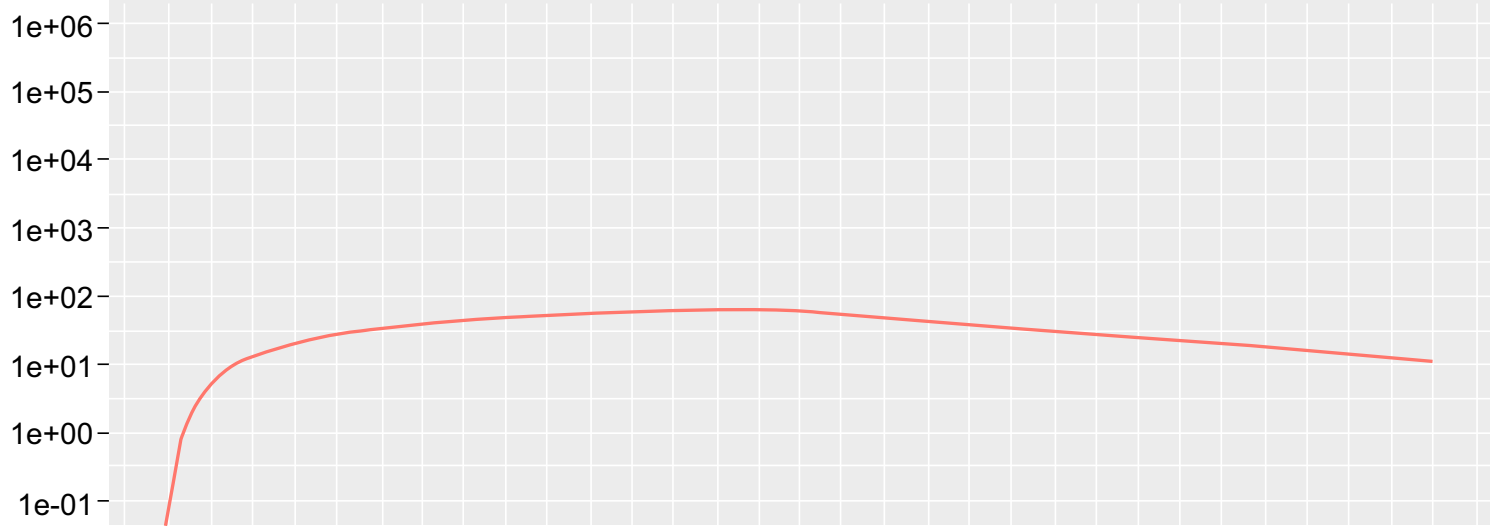

\section{Retina (macula)}

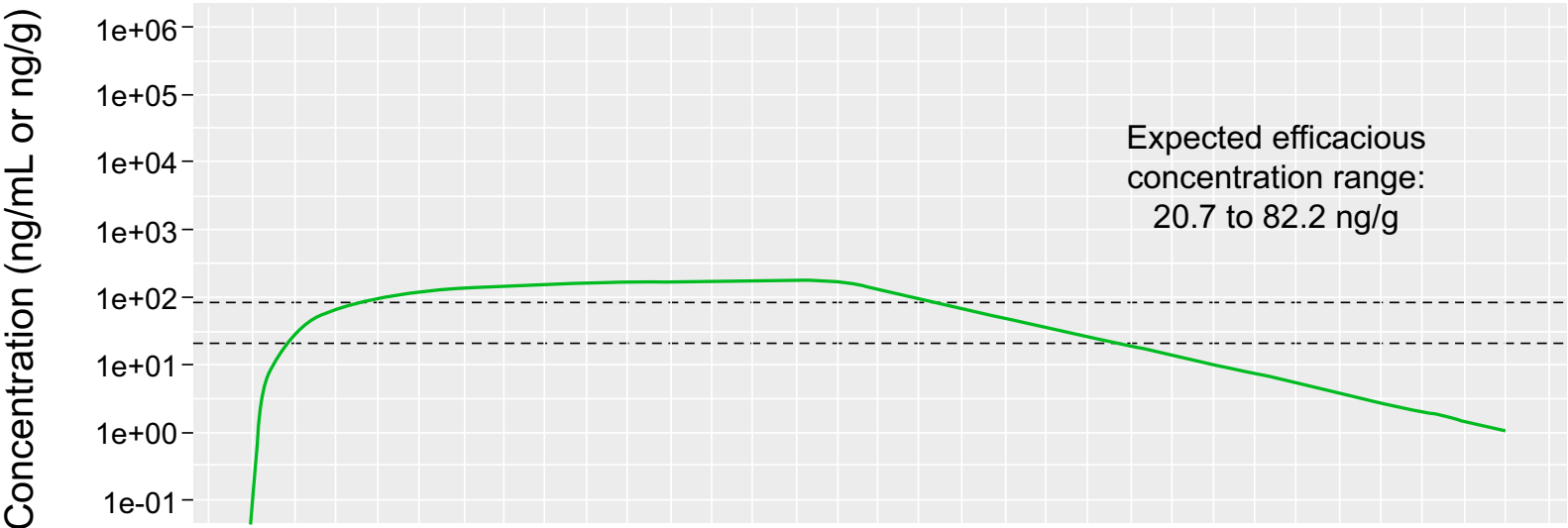

Vitreous

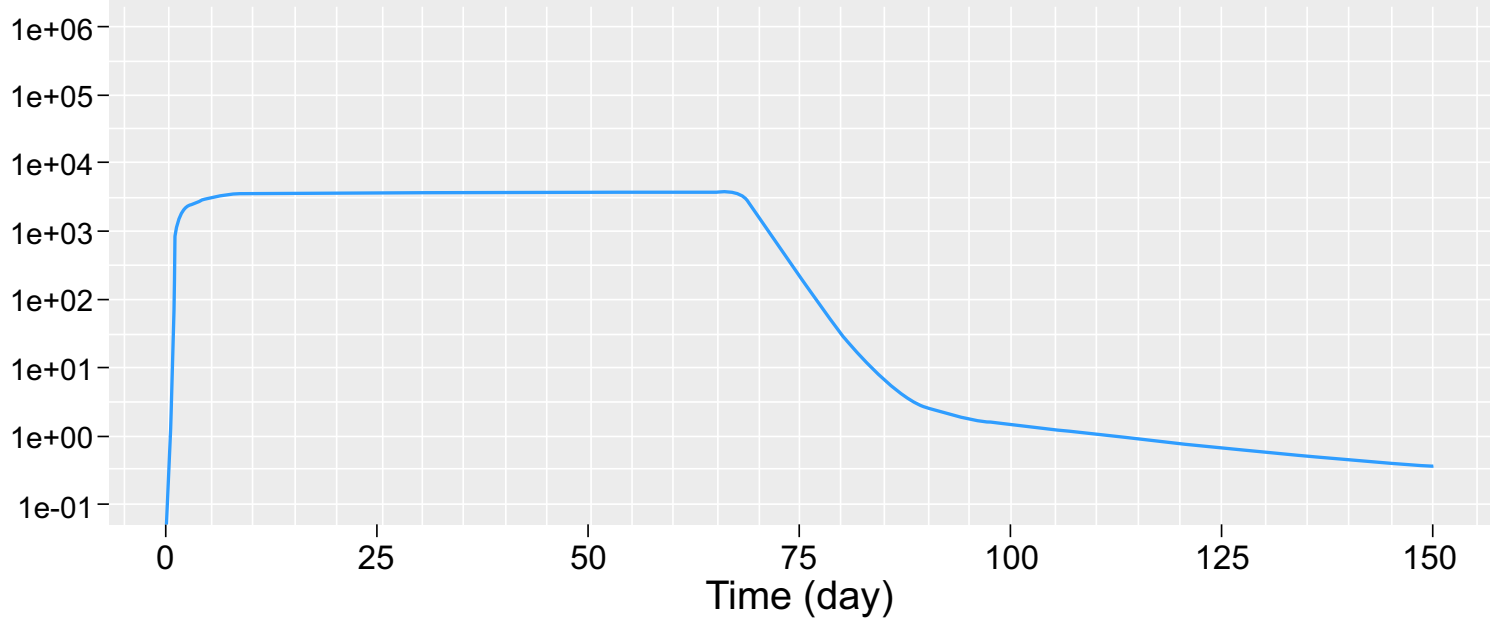

\section{Compartment}

\section{- Aqueous humor - Retina (macula) Vitreous}

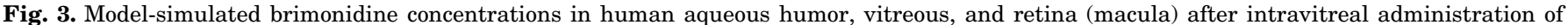

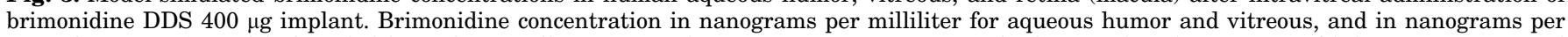

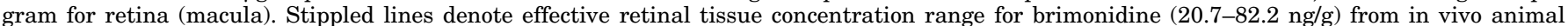
pharmacology model.

drug dose was evaluated with the optimized implant formulation because of the long duration of follow-up and the need to minimize the number of study animals. As studies with monkeys are resource-intensive, particularly those involving terminal procedures for collection of ocular tissues, we typically limit implant investigations to the final or near-final 
formulation. Although our linear pharmacokinetic model provided good data fit, the possibility of nonlinear pharmacokinetics should not be ruled out.

\section{Acknowledgments}

Medical writing and editorial assistance were provided to the authors by Andrew Fitton of Evidence Scientific Solutions (Horsham, UK) and funded by Allergan, an AbbVie company, Irvine, CA, USA. All authors met the ICMJE authorship criteria. No honoraria or payments were made for authorship.

\section{Authorship Contributions}

Participated in research design: Tamhane, Luu, Attar.

Conducted experiments: Tamhane.

Performed data analysis: Tamhane, Luu.

Wrote or contributed to the writing of the manuscript: Tamhane, Luu, Attar.

\section{References}

Acheampong AA, Shackleton M, John B, Burke J, Wheeler L, and Tang-Liu D (2002) Distribution of brimonidine into anterior and posterior tissues of monkey, rabbit, and rat eyes. Drug Metab Dispos 30:421-429.

Agrahari V, Mandal A, Agrahari V, Trinh HM, Joseph M, Ray A, Hadji H, Mitra R, Pal D, and Mitra AK (2016) A comprehensive insight on ocular pharmacokinetics. Drug Deliv Transl Res 6:735-754.

Bill A and Hellsing K (1965) Production and drainage of aqueous humor in the cynomolgus monkey (Macaca irus). Invest Ophthalmol 4:920-926.

Chang-Lin JE, Attar M, Acheampong AA, Robinson MR, Whitcup SM, Kuppermann BD, and Welty D (2011) Pharmacokinetics and pharmacodynamics of a sustainedrelease dexamethasone intravitreal implant. Invest Ophthalmol Vis Sci 52:80-86.

Del Amo EM, Rimpelä AK, Heikkinen E, Kari OK, Ramsay E, Lajunen T, Schmitt M Pelkonen L, Bhattacharya M, Richardson D, et al. (2017) Pharmacokinetic aspects of retinal drug delivery. Prog Retin Eye Res 57:134-185.

Del Amo EM and Urtti A (2015) Rabbit as an animal model for intravitreal pharmacokinetics: Clinical predictability and quality of the published data. Exp Eye Res 137:111-124.

Donello JE, Padillo EU, Webster ML, Wheeler LA, and Gil DW (2001) Alpha(2)-adrenoceptor agonists inhibit vitreal glutamate and aspartate accumulation and preserve retinal function after transient ischemia. J Pharmacol Exp Ther 296:216-223.

Feke GT, Tagawa H, Deupree DM, Goger DG, Sebag J, and Weiter JJ (1989) Blood flow in the normal human retina. Invest Ophthalmol Vis Sci 30:58-65.

Ferencz JR, Gilady G, Harel O, Belkin M, and Assia EI (2005) Topical brimonidine reduces collateral damage caused by laser photocoagulation for choroidal neovascularization. Graefes Arch Clin Exp Ophthalmol 243:877-880.

Galindo-Romero C, Harun-Or-Rashid M, Jiménez-López M, Vidal-Sanz M, AgudoBarriuso M, and Hallböö F (2016) Neuroprotection by $\alpha 2$-adrenergic receptor stimulation after excitotoxic retinal injury: a study of the total population of retinal ganglion cells and their distribution in the chicken retina. PLoS One 11:e161862.

Gaudreault J, Fei D, Rusit J, Suboc P, and Shiu V (2005) Preclinical pharmacokinetics of Ranibizumab (rhuFabV2) after a single intravitreal administration. Invest Ophthalmol Vis Sci 46:726-733.

Hernández M, Urcola JH, and Vecino E (2008) Retinal ganglion cell neuroprotection in a rat model of glaucoma following brimonidine, latanoprost or combined treatments. Exp Eye Res 86:798-806.

Kaufman PL, Calkins BT, and Erickson KA (1981) Ocular biometry of the cynomolgus monkey. Curr Eye Res 1:307-309.

Kent AR, Nussdorf JD, David R, Tyson F, Small D, and Fellows D (2001) Vitreous concentration of topically applied brimonidine tartrate $0.2 \%$. Ophthalmology 108:784-787.

Krupin T, Liebmann JM, Greenfield DS, Ritch R, and Gardiner S; Low-Pressure Glaucoma Study Group (2011) A randomized trial of brimonidine versus timolol in preserving visual function: results from the Low-Pressure Glaucoma Treatment Study. Am J Ophthalmol 151:671-681.

Kuppermann BD, Patel SS, Boyer DS, Augustin AJ, Freeman WR, Kerr KJ, Guo Q, Schneider S, and Lopez FJ (2020) Phase 2 study of the safety and efficacy of Brimonidine Drug Delivery System (Brimo DDS) Generation 1 in patients with geographic atrophy secondary to age-related macular degeneration. Retina. E-pub March 3.

Lafuente MP, Villegas-Pérez MP, Sobrado-Calvo P, García-Avilés A, Miralles de Imperial J, and Vidal-Sanz M (2001) Neuroprotective effects of alpha(2)-selective adrenergic agonists against ischemia-induced retinal ganglion cell death. Invest Ophthalmol Vis Sci 42:2074-2084.

Lai RK, Chun T, Hasson D, Lee S, Mehrbod F, and Wheeler L (2002) Alpha-2 adrenoceptor agonist protects retinal function after acute retinal ischemic injury in the rat. Vis Neurosci 19:175-185.

Merin S, Obolensky A, Farber MD, and Chowers I (2008) A pilot study of topical treatment with an alpha2-agonist in patients with retinal dystrophies. J Ocul Pharmacol Ther 24:80-86.
Mondal LK, Baidya KP, Bhattacharya B, Chatterjee PR, and Bhaduri G (2004) The efficacy of topical administration of brimonidine to reduce ischaemia in the very early stage of diabetic retinopathy in good controlled type-2 diabetes mellitus. $J$ Indian Med Assoc 102:724-725, 729.

Niwa Y, Kakinoki M, Sawada T, Wang X, and Ohji M (2015) Ranibizumab and aflibercept: intraocular pharmacokinetics and their effects on aqueous VEGF level in vitrectomized and nonvitrectomized Macaque eyes. Invest Ophthalmol Vis Sci 56:6501-6505.

Novack GD (2009) Ophthalmic drug delivery: development and regulatory considerations. Clin Pharmacol Ther 85:539-543.

Ortín-Martínez A, Valiente-Soriano FJ, García-Ayuso D, Alarcón-Martínez L, Jiménez-López M, Bernal-Garro JM, Nieto-López L, Nadal-Nicolás FM, VillegasPérez MP, Wheeler LA, et al. (2014) A novel in vivo model of focal light emitting diode-induced cone-photoreceptor phototoxicity: neuroprotection afforded by brimonidine, BDNF, PEDF or bFGF. PLoS One 9:e113798.

Peng M, Li Y, Luo Z, Liu C, Laties AM, and Wen R (1998) Alpha2-adrenergic agonists selectively activate extracellular signal-regulated kinases in Müller cells in vivo. Invest Ophthalmol Vis Sci 39:1721-1726.

Rajagopalan L, Ghosn C, and Tamhane M (2019) Cyto-/neuro-protective effect of brimonidine drug delivery system (DDS) in a nonhuman primate progressive retinal degeneration model of geographic atrophy (GA) secondary to age-related macular degeneration (AMD). Invest Ophthalmol Vis Sci 60:2993 Abstract.

Ramírez C, Cáceres-del-Carpio J, Chu J, Chu J, Moustafa MT, Chwa M, Limb GA, Kuppermann BD, and Kenney MC (2016) Brimonidine can prevent in vitro hydroquinone damage on retinal pigment epithelium cells and retinal Müller cells. $J$ Ocul Pharmacol Ther 32:102-108.

Saylor M, McLoon LK, Harrison AR, and Lee MS (2009) Experimental and clinical evidence for brimonidine as an optic nerve and retinal neuroprotective agent: an evidence-based review. Arch Ophthalmol 127:402-406.

Shen J, Durairaj C, Lin T, Liu Y, and Burke J (2014) Ocular pharmacokinetics of intravitreally administered brimonidine and dexamethasone in animal models with and without blood-retinal barrier breakdown. Invest Ophthalmol Vis Sci 55:1056-1066.

Shinno K, Kurokawa K, Kozai S, Kawamura A, Inada K, and Tokushige H (2017) The relationship of brimonidine concentration in vitreous body to the free concentration in retina/choroid following topical administration in pigmented rabbits. Curr Eye Res 42:748-753.

Short BG (2008) Safety evaluation of ocular drug delivery formulations: techniques and practical considerations. Toxicol Pathol 36:49-62.

Simó R, Hernández C, Porta M, Bandello F, Grauslund J, Harding SP, Aldington SJ, Egan C, Frydkjaer-Olsen U, García-Arumí J, et al.; European Consortium for the Early Treatment of Diabetic Retinopathy (EUROCONDOR) (2019) Effects of topically administered neuroprotective drugs in early stages of diabetic retinopathy: Results of the EUROCONDOR clinical trial. Diabetes 68:457-463.

Struble C, Howard S, and Relph J (2014) Comparison of ocular tissue weights (volumes) and tissue collection techniques in commonly used preclinical animal species. Poster presentation at European Association for Vision and Eye Research (EVER), Nice, France, October 1-4, 2014

Tamhane M, Robinson MR, and Attar M (2016) Ocular pharmacokinetics of brimonidine drug delivery system in rabbits and monkeys and its application in selecting dosing frequency in human clinical trials. Invest Ophthalmol Vis Sci 57:2154

Tang-Liu D, Acheampong A, Chien D-S, and Luo A (1992) The effect of melanin binding on brimonidine disposition in rabbit eyes. Invest Ophthalmol Vis Sci 33:1015 Abstract.

Valiente-Soriano FJ, Ortín-Martínez A, Di Pierdomenico J, García-Ayuso D, GallegoOrtega A, Miralles de Imperial-Ollero JA, Jiménez-López M, Villegas-Pérez MP, Wheeler LA, and Vidal-Sanz M (2019) Topical brimonidine or intravitreal BDNF, CNTF, or bFGF protect cones against phototoxicity. Transl Vis Sci Technol 8:36.

Varela-Fernández R, Díaz-Tomé V, Luaces-Rodríguez A, Conde-Penedo A, GarcíaOtero X, Luzardo-Álvarez A, Fernández-Ferreiro A, and Otero-Espinar FJ (2020) Drug delivery to the posterior segment of the eye: biopharmaceutic and pharmacokinetic considerations. Pharmaceutics 12:269.

Vidal-Sanz M, Lafuente MP, Mayor S, de Imperial JM, and Villegas-Pérez MP (2001) Retinal ganglion cell death induced by retinal ischemia. Neuroprotective effects of two alpha-2 agonists. Surv Ophthalmol 45 (Suppl 3):S261-S267, discussion S273-S276.

Weir AB and Collins M (2013) Assessing Ocular Toxicology in Laboratory Animals, Springer, New York.

Wheeler L, WoldeMussie E, and Lai R (2003) Role of alpha-2 agonists in neuroprotection. Surv Ophthalmol 48 (Suppl 1):S47-S51.

Wilhelm B, Lüdtke H, and Wilhelm H; BRAION Study Group (2006) Efficacy and tolerability of $0.2 \%$ brimonidine tartrate for the treatment of acute non-arteritic anterior ischemic optic neuropathy (NAION): a 3-month, double-masked, randomised, placebo-controlled trial. Graefes Arch Clin Exp Ophthalmol 244:551-558.

WoldeMussie E, Ruiz G, Wijono M, and Wheeler LA (2001) Neuroprotection of retinal ganglion cells by brimonidine in rats with laser-induced chronic ocular hypertension. Invest Ophthalmol Vis Sci 42:2849-2855.

Woldemussie E, Wijono M, and Pow D (2007) Localization of alpha 2 receptors in ocular tissues. Vis Neurosci 24:745-756.

Yoles E, Wheeler LA, and Schwartz M (1999) Alpha2-adrenoreceptor agonists are neuroprotective in a rat model of optic nerve degeneration. Invest Ophthalmol Vis Sci 40:65-73.

Address correspondence to: Dr. Mitalee Tamhane, AbbVie Inc., RD2-2B, 2525 Dupont Dr., Irvine, CA 92612. E-mail: mitalee.tamhane@abbvie.com 